PDES, SUBMANIFOLDS AND

AFFINE DIFFERENTIAL GEOMETRY

BANACH CENTER PUBLICATIONS, VOLUME 57

INSTITUTE OF MATHEMATICS

POLISH ACADEMY OF SCIENCES

WARSZAWA 2002

\title{
ON SOME TYPE OF CURVATURE CONDITIONS
}

\author{
MOHAMED BELKHELFA \\ Department of Mathematics, Katholieke Universiteit Leuven \\ Celestijnenlaan 200B, B-3001 Leuven, Belgium \\ E-mail: Mohamed.Belkhelfa@wis.kuleuven.ac.be \\ RYSZARD DESZCZ, MAŁGORZATA GŁOGOWSKA \\ Department of Mathematics, Agricultural University of Wrockaw \\ Grunwaldzka 53, 50-357 Wrockaw, Poland \\ E-mail: rysz@ozi.ar.wroc.pl,mglog@ozi.ar.wroc.pl \\ MARIAN HOTLOŚ \\ Institute of Mathematics, Wrockaw University of Technology \\ Wybrzeże Wyspiańskiego 27, 50-370 Wroctaw, Poland \\ E-mail:hotlos@im.pwr.wroc.pl \\ DOROTA KOWALCZYK \\ Department of Mathematics, Agricultural University of Wroctaw \\ Grunwaldzka 53, 50-357 Wrockaw, Poland \\ E-mail:dorotka@ozi.ar.wroc.pl \\ LEOPOLD VERSTRAELEN \\ Department of Mathematics, Katholieke Universiteit Leuven \\ Celestijnenlaan 200B, B-3001 Leuven, Belgium \\ E-mail: Leopold.Verstraelen@wis.kuleuven.ac.be
}

\footnotetext{
Abstract. In this paper we present a review of recent results on semi-Riemannian manifolds satisfying curvature conditions of pseudosymmetry type.

2000 Mathematics Subject Classification: Primary 53B20, 53B25; Secondary 53B50, 53C25, $53 \mathrm{C} 80$.

Key words and phrases: semisymmetric manifold, pseudosymmetry type curvature condition, generalized Einstein metric condition, warped product, Akivis-Goldberg metric, hypersurface, Cartan hypersurface, Chen's basic equality.

The second and fourth named authors are supported by the grant 2 P03A 00617 from the Polish State Committee for Scientific Research (KBN).

The paper is in final form and no version of it will be published elsewhere.
} 
1. Introduction. A semi-Riemannian manifold $(M, g), n \geq 3$, is said to be semisymmetric $([46])$ if

$$
R \cdot R=0
$$

on $M$. If on $M$ we have

$$
R \cdot S=0
$$

then $(M, g)$ is called Ricci-semisymmetric. The manifold $(M, g), n \geq 4$, is said to be Weyl-semisymmetric if

$$
R \cdot C=0
$$

on $M$. For precise definitions of the symbols used, we refer to Section 2 of this paper. We say that (1)-(3) are conditions of semisymmetric type ([16], Section 4.3). In Section 3 of this paper we present also other relations of this type. Some considerations on semiRiemannian manifolds satisfying the conditions of semisymmetric type lead to weaker curvature conditions, namely to pseudosymmetry type conditions (e.g., see [9], [16] and [48]). In this paper we present a review of recent results on semi-Riemannian manifolds satisfying such conditions. The basic class of manifolds related to this subject is formed by pseudosymmetric manifolds. A semi-Riemannian manifold $(M, g), n \geq 3$, is said to be pseudosymmetric ([16], Section 3.1; [48]) if at every point of $M$ the tensors $R \cdot R$ and $Q(g, R)$ are linearly dependent. Thus $(M, g)$ is pseudosymmetric if and only if on $U_{R}=\left\{x \in M \mid R-\frac{\kappa}{n(n-1)} G \neq 0\right.$ at $\left.x\right\}$ we have

$$
R \cdot R=L_{R} Q(g, R)
$$

where $L_{R}$ is some function on $U_{R}$. It is clear that every semisymmetric manifold is pseudosymmetric. The converse statement is not true. For instance, the Schwarzschild spacetime is a non-semisymmetric pseudosymmetric manifold ([30]). In Section 4 we present results on pseudosymmetric manifolds. In that section we give also results related to Ricci-pseudosymmetric, Weyl-pseudosymmetric manifolds as well as manifolds with pseudosymmetric Weyl tensor. We mention that every hypersurface in a Riemannian space of constant curvature realizing Chen's basic equality ([11]) is a manifold with pseudosymmetric Weyl tensor [31](Corollary 5.1).

In Section 5 we present other groups of conditions of pseudosymmetry type. A semiRiemannian manifold $(M, g), n \geq 3$, is said to be a Ricci-generalized pseudosymmetric manifold ([16], Section 5.3) if at every point of $M$ the tensors $R \cdot R$ and $Q(S, R)$ are linearly dependent. Thus $(M, g)$ is Ricci-generalized pseudosymmetric if and only if on $U=\{x \in M \mid Q(S, R) \neq 0$ at $x\}$ we have

$$
R \cdot R=L Q(S, R),
$$

where $L$ is some function on $U$. A Ricci-generalized pseudosymmetric manifold is called special if on $M$ we have

$$
R \cdot R=Q(S, R)
$$


The condition (6) arose during investigations of curvature properties of semi-Riemannian manifolds satisfying

$$
\omega(X) \mathcal{R}(Y, Z)+\omega(Y) \mathcal{R}(Z, X)+\omega(Z) \mathcal{R}(X, Y)=0,
$$

where $\omega$ is a 1 -form on $M$. Namely, it is known that (7) implies (6) on a subset of $M$ consisting of all points at which the 1 -form $\omega$ is nonzero ([16], Section 5.3). In Section 5 we present results on semi-Riemannian manifolds satisfying (6). Among other things, we state that this condition is fulfilled on every hypersurface $M$ of a semi-Euclidean space $\mathbb{E}_{s}^{n+1}$, with signature $(s, n+1-s)$. In other words, the difference tensor $R \cdot R-Q(S, R)$ vanishes on $M$. In the case when the ambient space is a semi-Riemannian space of constant curvature $N_{s}^{n+1}(c), n \geq 4$, with signature $(s, n+1-s)$, the tensor $R \cdot R-Q(S, R)$ of every hypersurface $M$ in $N_{s}^{n+1}(c)$ is expressed by (e.g., see [16], Section 5.5)

$$
R \cdot R-Q(S, R)=-\frac{(n-2) \widetilde{\kappa}}{n(n+1)} Q(g, C),
$$

where $\widetilde{\kappa}$ is the scalar curvature of the ambient space. The last relation leads to the next condition of pseudosymmetry type on a semi-Riemannian manifolds $(M, g)$ : at every point of $M$ the tensors $R \cdot R-Q(S, R)$ and $Q(g, C)$ are linearly dependent. This is equivalent on $U_{C}=\{x \in M \mid C \neq 0$ at $x\}$ to

$$
R \cdot R-Q(S, R)=L_{1} Q(g, C),
$$

where $L_{1}$ is some function on $U_{C}$. It is also known that any 4-dimensional warped product $M_{1} \times{ }_{F} M_{2}, \operatorname{dim} M_{1}=1$, fulfils (9) ([13]). Thus, in particular, every generalized RobertsonWalker spacetime ([4]) satisfies (9). There are also 4-dimensional non-warped product manifolds which realize (9). For instance, the Akivis-Goldberg metrics have this property. In Section 5 we describe in detail curvature properties of this class of metrics. In that section we also consider other curvature conditions of pseudosymmetry type. Among other things we present conditions related to hypersurfaces in spaces of constant curvature or in affine hypersurfaces ([6], [15], [17] and [20]).

In Section 6 we present results on quasi-Einstein hypersurfaces in $\mathbb{E}_{s}^{n+1}(c), n \geq 4$, satisfying some curvature condition of pseudosymmetry type (see [21], [26], [28]). These results are closely related to the warped product solutions of the P. J. Ryan problem (see [19] and [20] and references therein).

Recently, in [27] a curvature property of pseudosymmetry type of Einstein manifolds has been found. Namely we have

THEOREM 1.1 ([27], Theorem 3.1). On any semi-Riemannian Einstein manifold the following identity is satisfied:

$$
R \cdot C-C \cdot R=\frac{\kappa}{(n-1) n} Q(g, R)=\frac{\kappa}{(n-1) n} Q(g, C) .
$$

Evidently, for $n=2$ or $n=3$, the above condition is satisfied trivially. Motivated by the above theorem we introduced in [27] (see also [24]) a family of curvature conditions of pseudosymmetry type. In Section 7 we present results on non-Einstein and non-conformally flat semi-Riemannian manifolds fulfilling these conditions. 
We finish this section with a remark that every condition of pseudosymmetry type on a semi-Riemannian manifold $(M, g)$ is on a certain open subset of $M$ locally equivalent to a system of partial differential equations of second order.

2. Basic definitions. In this section we present definitions of the tensors considered in this paper. Let $(M, g)$ be an $n$-dimensional, $n \geq 3$, semi-Riemannian connected manifold of class $C^{\infty}$. We denote by $\nabla, S$ and $\kappa$, the Levi-Civita connection, the Ricci tensor and the scalar curvature of $(M, g)$, respectively. We define on $M$ the endomorphisms $X \wedge_{A} Y, \mathcal{R}(X, Y)$ and $\mathcal{C}(X, Y)$ by

$$
\begin{aligned}
\left(X \wedge_{A} Y\right) Z & =A(Y, Z) X-A(X, Z) Y \\
\mathcal{R}(X, Y) Z & =\nabla_{X} \nabla_{Y} Z-\nabla_{Y} \nabla_{X} Z-\nabla_{[X, Y]} Z \\
\mathcal{C}(X, Y) & =\mathcal{R}(X, Y)-\frac{1}{n-2}\left(X \wedge_{g} \mathcal{S} Y+\mathcal{S} X \wedge_{g} Y-\frac{\kappa}{n-1} X \wedge_{g} Y\right)
\end{aligned}
$$

respectively, where $A$ is a $(0,2)$-tensor on $M, X, Y, Z \in \Xi(M), \Xi(M)$ being the Lie algebra of vector fields on $M$ and the Ricci operator $\mathcal{S}$ is defined by $g(X, \mathcal{S} Y)=$ $S(X, Y)$. The Riemann curvature tensor $R$, the Weyl tensor $C$ and the $(0,4)$-tensor $G$ of $(M, g)$ are defined by $R\left(X_{1}, X_{2}, X_{3}, X_{4}\right)=g\left(\mathcal{R}\left(X_{1}, X_{2}\right) X_{3}, X_{4}\right), C\left(X_{1}, X_{2}, X_{3}, X_{4}\right)=$ $g\left(\mathcal{C}\left(X_{1}, X_{2}\right) X_{3}, X_{4}\right)$ and $G\left(X_{1}, X_{2}, X_{3}, X_{4}\right)=g\left(\left(X_{1} \wedge_{g} X_{2}\right) X_{3}, X_{4}\right)$, respectively. For symmetric $(0,2)$-tensors $A$ and $B$ we define their Kulkarni-Nomizu product $A \wedge B$ by

$$
\begin{aligned}
(A \wedge B)\left(X_{1}, X_{2} ; X, Y\right)= & A\left(X_{1}, Y\right) B\left(X_{2}, X\right)+A\left(X_{2}, X\right) B\left(X_{1}, Y\right) \\
& -A\left(X_{1}, X\right) B\left(X_{2}, Y\right)-A\left(X_{2}, Y\right) B\left(X_{1}, X\right) .
\end{aligned}
$$

Let $\mathcal{B}(X, Y)$ be a skew-symmetric endomorphism of $\Xi(M)$ and let $B$ be a $(0,4)$-tensor associated with $\mathcal{B}(X, Y)$ by

$$
B\left(X_{1}, X_{2}, X_{3}, X_{4}\right)=g\left(\mathcal{B}\left(X_{1}, X_{2}\right) X_{3}, X_{4}\right) .
$$

It is well known that the tensor $B$ is said to be a generalized curvature tensor if the following conditions are fulfilled:

$$
\begin{aligned}
& B\left(X_{1}, X_{2}, X_{3}, X_{4}\right)+B\left(X_{2}, X_{3}, X_{1}, X_{4}\right)+B\left(X_{3}, X_{1}, X_{2}, X_{4}\right)=0, \\
& B\left(X_{1}, X_{2}, X_{3}, X_{4}\right)=B\left(X_{3}, X_{4}, X_{1}, X_{2}\right)=0 .
\end{aligned}
$$

Clearly, the the tensors $R, C$ and $G$ are generalized curvature tensors. It is also easy to see that $A \wedge B$ is also a generalized curvature tensor.

Let $\mathcal{B}(X, Y)$ be a skew-symmetric endomorphism of $\Xi(M)$ and let $B$ be the tensor defined by (11). We extend the endomorphism $\mathcal{B}(X, Y)$ to a derivation $\mathcal{B}(X, Y)$. of the algebra of tensor fields on $M$, assuming that it commutes with contractions and $\mathcal{B}(X, Y)$. $f=0$ for any smooth function on $M$. Now for a $(0, k)$-tensor field $T, k \geq 1$, we can define the $(0, k+2)$-tensor $B \cdot T$ by

$$
\begin{aligned}
& (B \cdot T)\left(X_{1}, \ldots, X_{k} ; X, Y\right)=(\mathcal{B}(X, Y) \cdot T)\left(X_{1}, \ldots, X_{k} ; X, Y\right) \\
= & -T\left(\mathcal{B}(X, Y) X_{1}, X_{2}, \ldots, X_{k}\right)-\cdots-T\left(X_{1}, \ldots, X_{k-1}, \mathcal{B}(X, Y) X_{k}\right) .
\end{aligned}
$$

In addition, if $A$ is a symmetric $(0,2)$-tensor field then we define the $(0, k+2)$-tensor 
$Q(A, T)$ by

$$
\begin{aligned}
& Q(A, T)\left(X_{1}, \ldots, X_{k} ; X, Y\right)=\left(X \wedge_{A} Y \cdot T\right)\left(X_{1}, \ldots, X_{k} ; X, Y\right) \\
= & -T\left(\left(X \wedge_{A} Y\right) X_{1}, X_{2}, \ldots, X_{k}\right)-\cdots-T\left(X_{1}, \ldots, X_{k-1},\left(X \wedge_{A} Y\right) X_{k}\right) .
\end{aligned}
$$

In particular, in this way, we obtain the $(0,6)$-tensors $B \cdot B$ and $Q(A, B)$. Setting in the above formulas $\mathcal{B}=\mathcal{R}$ or $\mathcal{B}=\mathcal{C}, T=R$ or $T=C$ or $T=S, A=g$ or $A=S$, we get the tensors $R \cdot R, R \cdot C, C \cdot R, C \cdot C, Q(g, R), Q(g, C), Q(S, G), Q(S, R), Q(S, C), R \cdot S$, $C \cdot S$, and $Q(g, S)$.

For a $(0, k)$-tensor field $T, k \geq 1$, and a symmetric $(0,2)$-tensor field $A$ on $M$, we define the $(0, k)$-tensor $A \cdot T$ by

$$
(A \cdot T)\left(X_{1}, \ldots, X_{k}\right)=-T\left(\mathcal{A} X_{1}, X_{2}, \ldots, X_{k}\right)-\ldots-T\left(X_{1}, X_{2}, \ldots, \mathcal{A} X_{k}\right),
$$

where the endomorphism $\mathcal{A}$ is defined by $g(\mathcal{A} X, Y)=A(X, Y)$. Putting in the above formula $T=R$ or $T=C$ and $A=S$, we obtain the tensors: $S \cdot R$ and $S \cdot C$.

Let $M$ be a hypersurface in a semi-Riemannian space of constant curvature $N_{s}^{n+1}(c)$, $n \geq 4$. Let $g$ denote the metric tensor of $M$ induced from the metric tensor of $N_{s}^{n+1}(c)$. The second fundamental tensor $H$ and the shape operator $\mathcal{A}$ of $M$ satisfy the relation $H^{k}(X, Y)=g\left(\mathcal{A}^{k} X, Y\right), k=1,2, \ldots$, where $X, Y$ are vector fields tangent to $M$. We denote by $U_{H}$ the subset of $M$ consisting of all points at which $H^{2}$ is not a linear combination of the tensors $H$ and $g$.

3. Semisymmetric manifolds. It is well known that the class of semisymmetric manifolds includes the set of locally symmetric manifolds $(\nabla R=0)$ as a proper subset. Semisymmetric Riemannian manifolds were first studied by E. Cartan, A. Lichnerowicz, R. S. Couty and N. S. Sinjukov. In [43] K. Nomizu asked whether there exist complete, irreducible and simply connected Riemannian manifolds of dimension $\geq 3$ satisfying (1) and not locally symmetric. The first positive example was constructed by H. Takagi ([47]). A fundamental study on Riemannian semisymmetric manifolds was made by Z. I. Szabó (e.g., see [46]). Semisymmetric non-conformally flat $(C \neq 0)$ semi-Riemannian manifolds were studied by A. Derdziński and W. Roter. They investigated semisymmetric manifolds having parallel Weyl tensor $(\nabla C=0)$ as well as semisymmetric manifolds with recurrent Weyl tensor $(\nabla C=C \otimes \psi)$ (see [44] and references therein). Under some additional assumptions such manifolds satisfy (9). In particular, we have

Theorem 3.1 (cf. [23], Theorem 4.3). Every manifold $(M, g), n \geq 4$, with parallel Weyl tensor, which is nor locally symmetric and nor conformally flat, satisfies (9).

Recently, semisymmetric semi-Riemannian manifolds were investigated in [38] and [42]. In particular, in [38] there have been investigated curvature properties of pseudosymmetry type of semi-Riemannian manifolds satisfying the following condition:

$$
R=\frac{\phi}{2} S \wedge S
$$

on the set $U \subset M$ consisting of all points of $M$ at which $\operatorname{rank} S>1$, where $\phi$ is some function on $U$. 
We note that the class of Ricci-semisymmetric manifolds includes the set of Riccisymmetric manifolds $(\nabla S=0)$ as a proper subset. Every semisymmetric manifold is Ricci-semisymmetric. The converse statement is not true. However, under some additional assumptions, (1) and (2) are equivalent. The problem of the equivalence of (1) and (2) was considered by several authors (e.g., see [1], [5], [19] and [20] and references therein). Recently, examples of non-semisymmetric quasi-Einstein Ricci-symmetric hypersurfaces in Euclidean spaces $\mathbb{E}^{n+1}, n \geq 5$, were found in [1]. There also exist non-semisymmetric Ricci-symmetric (quasi-Einstein or non-quasi-Einstein) hypersurfaces in semi-Euclidean spaces $\mathbb{E}_{s}^{n+1}, n \geq 5$, ([19], see also [20]). In Section 6 we present results on quasi-Einstein hypersurfaces in semi-Euclidean spaces.

Every semisymmetric manifold is Weyl-semisymmetric. The converse statement is not true. It is known that (1) and (3) are equivalent on the set $U_{C}$ of any semi-Riemannian manifold $(M, g)$ of dimension $n \geq 5$. If $n=4$ this statement is not true. There exists a 4 dimensional non-semisymmetric and non-conformally flat Riemannian manifold satisfying (3). We refer to [16](Section 9) for a review of results related to this subject.

There also exist semi-Riemannian manifolds which satisfy the following condition (e.g., see [16], Section 5.6):

$$
\omega(X) \mathcal{C}(Y, Z)+\omega(Y) \mathcal{C}(Z, X)+\omega(Z) \mathcal{C}(X, Y)=0,
$$

where $\omega$ is a 1 -form on $M$. If this is satisfied at a point $x \in M$ and the 1-form $\omega$ is nonzero at this point, then at $x$ we have $([16]$, Section 5.6) $C \cdot C=0$. Recently, semi-Riemannian manifolds satisfying other curvature conditions of semisymmetry type: $S \cdot R=0$ or

$$
S \cdot C=0
$$

were investigated e.g. in: [12], [21] and [34].

4. Pseudosymmetric manifolds. The condition (4) arose during the study on totally umbilical submanifolds of semisymmetric manifolds ([16], Section 13), as well as when considering geodesic mappings of semisymmetric manifolds ([16], Section 10). It is known that every totally umbilical submanifold, with parallel mean curvature vector field, in a semi-Riemannian semisymmetric (or more generally, in a pseudosymmetric) manifold is also pseudosymmetric. In particular, extrinsic spheres in pseudosymmetric Riemannian manifolds are also pseudosymmetric. Further, if a semi-Riemannian manifold $(M, g)$ admits a geodesic mapping onto a semisymmetric (or more general, onto a pseudosymmetric) manifold then $(M, g)$ is pseudosymmetric. The class of pseudosymmetric manifolds is the widest known class of manifolds which is closed with respect to geodesic mappings. It is easy to verify that (4) is invariant under concircular deformations ([16], Section 11). We also mention that the metric of the Schwarzschild spacetime is a conformal deformation of some semisymmetric metric. The Schwarzschild spacetime, the Kottler spacetime as well as the Reissner-Nordström spacetime are nonsemisymmetric pseudosymmetric manifolds ([14], [30]). In general, the Robertson-Walker spacetimes are pseudosymmetric. However, in some special case, certain RobertsonWalker spacetimes are semisymmetric ([16], Section 6). For more detailed information on the geometric motivation for the introduction of pseudosymmetric manifolds, and for 
a review of results on different aspects of pseudosymmetric manifolds we refer to [16] and $[48]$.

Let $\mathcal{S}$ be the Ricci operator of a semi-Riemannian manifold $(M, g)$. It is known that at every point $x$ of a conformally flat semisymmetric semi-Riemannian manifold $(M, g)$ its operator $\mathcal{S}^{2}$ is a linear combination of $\mathcal{S}$ and the identity transformation of $T_{x} M$. The converse statement is not true. If at every point of a conformally flat manifold $(M, g)$ its Ricci operator has the above property then, in general, $(M, g)$ is a non-semisymmetric pseudosymmetric manifold ([16], Section 6). We mention that there also exist non-conformally flat pseudosymmetric manifolds which are non-semisymmetric (e.g., see [16], Section 3.6).

A special subclass of pseudosymmetric manifolds is determined by the following decomposition of the curvature tensor of $(M, g)$.

THEOREM 4.1 (cf. [23], Theorem 4.2). Let $(M, g), n \geq 4$, be a semi-Riemannian manifold. If on the set $U_{S} \cap U_{C} \subset M$ its curvature tensor $R$ has the decomposition

$$
R=\frac{\phi}{2} S \wedge S+\mu g \wedge S+\eta G,
$$

then on this set we have (4) and

$$
R \cdot R=Q(S, R)+\left(L_{R}+\frac{\mu}{\phi}\right) Q(g, C), \quad L_{R}=\frac{\mu}{\phi}((n-2) \mu-1)-\eta(n-2),
$$

where $\phi, \mu$ and $\eta$ are some functions on $U_{S} \cap U_{C}$.

We note that (12) is a special case of (14). Evidently, the Schwarzschild spacetime the Kottler spacetime as well as the Robertson-Walker spacetimes can not fullfil (14). In [39](Example 5.3) a class of four dimensional warped products satisfying (14) were determined. In particular, in view of this result, we can state that the Reissner-Nordström spacetime fulfils (14) ([39]). We mention that in [39] generalized curvature tensors having a decomposition of the form (14) were investigated.

A pseudosymmetric manifold $(M, g), n \geq 3$, is said to be a pseudosymmetric manifolds of constant type if the function $L_{R}$, defined by (4) is constant on $U_{R}$ ([40], [41]). [36], [40] and [41] contain results related to local classification of 3-dimensional pseudosymmetric manifolds of constant type.

Recently, some results on pseudosymmetric D'Atri spaces of dimension $n=3$ or $n=4$ were obtained in [8] and [9] (see also [7]).

A semi-Riemannian manifold $(M, g)$ is said to be Ricci-pseudosymmetric ([16], Section 4.1 ) if at every point of $M$ the tensors $R \cdot S$ and $Q(g, S)$ are linearly dependent. Thus $(M, g)$ is Ricci-pseudosymmetric if and only if on $U_{S}=\left\{x \in M \mid S-\frac{\kappa}{n} g \neq 0\right.$ at $\left.x\right\}$ we have

$$
R \cdot S=L_{S} Q(g, S),
$$

where $L_{S}$ is some function on $U_{S}$. Note that $U_{S} \subset U_{R}$. If at a point $x$ of a manifold $(M, g)$ the condition (4) is satisfied then (15) holds at $x$. The converse statement is not true (e.g., see [16], Section 8). A family of compact non-pseudosymmetric Ricci-pseudosymmetric manifolds was described in [29].

The Cartan hypersurfaces in the sphere $S^{n+1}(c)$ are compact, minimal hypersurfaces with constant principal curvatures $-\sqrt{3 c}, 0, \sqrt{3 c}$ of the same multiplicity. Thus such 
hypersurfaces are isoparametric. The Cartan hypersurfaces exist only for $n=3,6,12$ or 24. More precisely, the Cartan hypersurfaces are tubes of constant radius over the standard Veronese embeddings $i: \mathbb{F} P^{2} \rightarrow S^{3 d+1}(c) \rightarrow \mathbb{E}^{3 d+2}, d=1,2,4$ or 8 , of the projective plane $\mathbb{F} P^{2}$ in the sphere $S^{3 d+1}(c)$ in $\mathbb{E}^{3 d+2}$, where $\mathbb{F}=\mathbb{R}$ (real numbers), $\mathbb{C}$ (complex numbers), $\mathbb{H}$ (quaternions) or $\mathbb{O}$ (octonions), respectively. These hypersurfaces were discovered by E. Cartan in his work about isoparametric hypersurfaces. Cartan hypersurfaces are manifolds, with non-parallel Ricci tensor, satisfying

$$
(\nabla S)(X, Y ; Z)+(\nabla S)(Y, Z ; X)+(\nabla S)(Z, X ; Y)=0
$$

for all $X, Y, Z \in \Xi(M)$. Further, in [32](Theorem 1) it was shown that every Cartan hypersurface in $S^{n+1}(c), n=6,12$ or 24 , is a non-pseudosymmetric Ricci-pseudosymmetric manifold fulfilling

$$
R \cdot S=\frac{\tau}{n(n+1)} Q(g, S),
$$

where $\tau$ is the scalar curvature of $S^{n+1}(c)$. Cartan hypersurface in $S^{4}(c)$ is a pseudosymmetric manifold satisfying $R \cdot R=\frac{\tau}{12} Q(g, R)$. Recently, new curvature properties of the Cartan hypersurfaces has been obtained in [20]. We present it in the next section.

We also mention that in [18](see also references therein) the conditions of pseudosymmetry and Ricci-pseudosymmetry realized on hypersurfaces of semi-Riemannian spaces of constant curvature were investigated. For instance, we have

Theorem 4.2 ([18], Theorems 3.1 and 5.1). Let $M$ be a hypersurface in $N_{s}^{n+1}(c)$, $n \geq 4$. Then $M$ is pseudosymmetric if and only if at every $x \in M$ its second fundamental tensor $H$ is of rank two or at this point $H^{2}$, the square of $H$, is a linear combination of $H$ and the metric tensor $g$. Moreover, on $U_{H} \subset M$ we have

$$
R \cdot R=\frac{\tau}{n(n+1)} Q(g, R) .
$$

THEOREM 4.3 (cf. [39], Example 5.4). The curvature tensor $R$ of a pseudosymmetric hypersurface $M$ in $N_{s}^{n+1}(c), n \geq 4$, is of the form (14) on the subset $U_{H} \subset M$.

Let $N^{2}(c)$ be a minimal surface with constant curvature $c$ in the unit $(n+1)$-sphere $S^{n+1}(1)$. According to [11](Section 6) the tubular hypersurface $T_{\frac{\pi}{2}}\left(N^{2}(c)\right)$ with radius $\frac{\pi}{2}$ about $N^{2}(c)$ is called a generalized Cartan hypersurface in $S^{n+1}(1)$. At every point of that hypersurface its second fundamental tensor $H$ fulfils $\operatorname{rank} H \leq 2$. Generalized Cartan hypersurfaces satisfy Chen's basic equality. This, together with Theorem 4.3, leads immediately to the following

COROLlary 4.1. Every generalized Cartan hypersurface, with $c \neq 0$, fulfils (18).

A semi-Riemannian manifold $(M, g), n \geq 4$, is said to be Weyl-pseudosymmetric ([16], Section 4.2) if at every point of $M$ the tensors $R \cdot C$ and $Q(g, C)$ are linearly dependent. Thus $(M, g)$ is Weyl-pseudosymmetric if and only if on $U_{C} \subset M$ we have

$$
R \cdot C=L_{C} Q(g, C),
$$

where $L_{C}$ is some function on $U_{C}$. Note that $U_{C} \subset U_{R}$. Evidently, every pseudosymmetric manifold is Weyl-pseudosymmetric. The converse statement is not true. It is known that (4) and (19) are equivalent on the subset $U_{C}$ of every semi-Riemannian manifold of 
dimension $n \geq 5$ (e.g., see [16], Section 9.2). Further, both conditions are equivalent on the subset $U_{C}$ of every 4-dimensional warped product manifold ([16], Section 9.3). Every conformally flat Riemannian manifold of dimension $\geq 4$ is Weyl-pseudosymmetric. There exist non-pseudosymmetric conformally flat manifolds (e.g., see [16], Section 3.5) as well as non-pseudosymmetric Weyl-pseudosymmetric manifolds with nonzero tensor $R \cdot C$ (e.g., see [16], Section 9.2).

A semi-Riemannian manifold $(M, g), n \geq 4$, is said to be a manifold with pseudosymmetric Weyl tensor ([16], Section 12.6) if at every point of $M$ the tensors $C \cdot C$ and $Q(g, C)$ are linearly dependent. Thus $(M, g)$ is a manifold with pseudosymmetric Weyl tensor if and only if

$$
C \cdot C=L Q(g, C),
$$

on $U_{C}$, where $L$ is some function on $U_{C}$. It is known that every warped product $M_{1} \times_{F} M_{2}$, $\operatorname{dim} M_{1}=\operatorname{dim} M_{2}=2$, fulfils (20) ([16], Section 12.6). Manifolds fulfilling (4) and (20) were investigated in [33]. Hypersurfaces with pseudosymmetric Weyl tensor were investigated e.g. in [31]. We have

TheOREM 4.4 ([31], Theorem 3.1). Any 2-quasi umbilical hypersurface, of dimension $n \geq 4$, in a conformally flat space is a manifold with pseudosymmetric Weyl tensor.

5. Other conditions of pseudosymmetry type. We recall that a Ricci-generalized pseudosymmetric manifold is called special if (6) holds on $M$. For results on manifolds satisfying (6) we refer to Section 5 of [16] and references therein. Recently, 4-dimensional warped products fulfilling (6) and (13) were studied in [12].

The warped product $M_{1} \times_{F} M_{2}$, of a 1-dimensional manifold $\left(M_{1}, \bar{g}\right), \bar{g}_{11}=-1$, with a warping function $F$, and a 3 -dimensional Riemannian manifold $\left(M_{2}, \widetilde{g}\right)$ is said to be a generalized Robertson-Walker spacetime (e.g., see [4]). Recently these spacetimes have been widely and intensively studied; see for example [45] and references therein. In particular, when the manifold $\left(M_{2}, \widetilde{g}\right)$ is a Riemannian space of constant curvature, the warped product $M_{1} \times_{F} M_{2}$ is called a Robertson-Walker spacetime. Every RobertsonWalker spacetime is pseudosymmetric (e.g., see [16], Section 12.2). In [13] it was shown that generalized Robertson-Walker spacetimes fulfil (9).

Recently, in [34] it was proved that 4-dimensional semi-Riemannian manifolds investigated in [3] also fulfil (9). Let $M$ be a manifold of dimension $n=p q$, and let $S C(p, q)$ be a differentiable field of Segre cones $S C_{x}(p, q) \subset T_{x} M, x \in M$. The pair $(M, S C(p, q))$ is called an almost Grassmann structure and is denoted by $A G(p-1, p+q-1)$. The manifold $M$ endowed with such structure is said to be an almost Grassmann manifold ([2], Chapter 7; [3], Definition 1.1). Certain additional conditions lead to so-called semiintegrable almost Grassmann structures ([3], Definition 1.2). The latter were studied in [3] and examples of such structures, mainly 4-dimensional, are presented there. Some semi-Riemannian metrics are related to these structures. The problem arises to describe curvature properties and, in particular, curvature properties of pseudosymmetry type, of these metrics. In [34] curvature properties of 4-dimensional metrics considered in the Examples 3.5 - 3.16 of [3] were found. These metrics will be called Akivis-Goldberg, in 
short $A G$-metrics. It is easy to check that the Ricci tensor $S$ and the scalar curvature $\kappa$ of an $A G$-metric satisfy

$$
\text { (i) } \quad \operatorname{rank} S \leq 2, \quad(\text { ii }) \quad S^{2}=0, \quad(\text { iii }) \quad \kappa=0 .
$$

In [34] it was shown that every $A G$-metric fulfils (13). We can also check that the $A G$ metrics considered in the Examples 3.5-3.13 and 3.15 of [3] satisfy (7) for some nonzero 1-form. Thus we see that these metrics fulfil also (6). In addition, in [34] it was shown that the $A G$-metric, defined in Example 3.5 of [3], is a non-warped product metric. This metric cannot be realized as a hypersurface in a space $N_{s}^{5}([34])$.

We denote by $\mathcal{C}$ the class of all semi-Riemannian manifolds, of dimensions $\geq 4$, satisfying (6). In this class we can distinguish at least three subclasses: $\mathcal{C}_{1}, \mathcal{C}_{2}$, and $\mathcal{C}_{3}$. The subclass $\mathcal{C}_{1}$ is formed by warped products ([16], Section 5.3). The subclass $\mathcal{C}_{2}$ is formed by semi-Riemannian manifolds whose curvature tensor $R$ satisfies $R=\varepsilon A \wedge A, \varepsilon= \pm 1$, for some symmetric $(0,2)$-tensor $A$. In particular, every hypersurface in $\mathbb{E}_{s}^{n+1}, n \geq 3$, belongs to this subclass $([16]$, Section 5.4$)$. The subclass $\mathcal{C}_{3}$ is formed by manifolds fulfilling (7) such that $\omega$ is nonzero on a dense subset of a given manifold. The $A G$-metric $g$ defined in Example 3.5 of [3] belongs to the subclass $\mathcal{C}_{3}$ but belongs neither to $\mathcal{C}_{1}$ nor to $\mathcal{C}_{2}$. This shows that the subclass $\mathcal{C}_{3}$ is essential. In [12] we present an example of a warped product manifold which belongs to the class $\mathcal{C}_{2}$ and does not belong to the class $\mathcal{C}_{3}$. It is an open problem whether there exist other subclasses of the class $\mathcal{C}$. The $A G$-metrics defined in the Examples 3.14 and 3.16 of [3] are semisymmetric metrics with a nonzero tensor $Q(S, R)$. Therefore both metrics do not satisfy (6). However, they satisfy (9). Thus we can state that the $A G$-metrics satisfy (9) ([34], Theorem 4.4). We recall that conformally symmetric manifolds which are neither locally symmetric and neither conformally flat also satisfy (9) ([23], Theorem 4.3). However, they form two disjoint subclasses of the class of all semi-Riemannian manifolds satisfying (9) ([34], Remark 4.9).

[22] considers semi-Riemannian manifolds $(M, g), n \geq 4$, fulfilling at every point the condition: the tensors $R \cdot C$ and $Q(S, C)$ are linearly dependent. This is equivalent on the set $U=\{x \in M \mid Q(S, C) \neq 0$ at $x\}$ to

$$
R \cdot C=L Q(S, C),
$$

where $L$ is some function on $U$. We note that every semisymmetric manifold, as well as every Weyl-semisymmetric manifold, satisfies (22) trivially. There also exist nonsemisymmetric and non-Weyl-semisymmetric manifolds satisfying this condition. Manifolds satisfying (22) have recently been investigated e.g. in: [21], [22], [25], [26] and [28]. The conditions: (4), (5), (9), (15), (19), (20) and (22) are called conditions of pseudosymmetry type ([15], [16] and [48]).

As mentioned in the previous section, new curvature properties of the Cartan hypersurfaces of dimension $n=6,12$ or 24 have been obtained in [20]. Namely, we have

Theorem 5.1 (cf. [20], Theorem 4.3). On every Cartan hypersurface $M$ in $S^{n+1}(c)$, $n=6,12$ or 24 , the following relations are fulfilled: (17),

$$
R \cdot C=Q(S, R)-\frac{(n-2) \tau}{n(n+1)} Q(g, R)-\frac{(n-3) \tau}{(n-2) n(n+1)} Q(S, G),
$$




$$
\begin{aligned}
C \cdot R & =\frac{n-3}{n-2} Q(S, R)-\frac{(n-3) \tau}{(n-1)(n+1)} Q(g, R)-\frac{(n-3) \tau}{(n-2) n(n+1)} Q(S, G), \\
C \cdot C & =\frac{n-3}{n-2} Q(S, R)-\frac{(n-3) \tau}{(n-1)(n+1)} Q(g, R)-\frac{(n-3)\left(n^{2}-n-3\right) \tau}{(n-2)^{2} n(n+1)^{2}} Q(S, G) .
\end{aligned}
$$

Very recently, hypersurfaces of semi-Riemannian spaces of constant curvature $N_{s}^{n+1}(c), n \geq 4$, satisfying (23) were investigated in [35]. Among other things, the following curvature characterization of Ricci-pseudosymmetric hypersurfaces was proved.

Theorem 5.2 ([35], Theorem 6.1). Let $M$ be a hypersurface in $N_{s}^{n+1}, n \geq 4$. Then (17) is fulfilled on $U_{H} \subset M$ if and only if (23) holds on $U_{H}$.

There are also other curvature conditions of pseudosymmetry type. For instance, a family of conditions of pseuodsymmetry type on non-degenerate affine hypersurfaces $M$ were introduced and investigated in [15] and [17]. These papers investigated curvature conditions formed with the tensors $R^{*} \cdot R^{*}, Q\left(\operatorname{Ric}\left(R^{*}\right), R^{*}\right), Q\left(h, R^{*}\right)$, where $R^{*}$ is the Opozda-Verstraelen curvature tensor and $h$ is the Blaschke-Berwald metric on $M$. We also mention that recently a group of conditions of pseudosymmetry type formed by the tensors: $R, C$ and powers $H^{k}, k=1,2,3$, obtained from the second fundamental tensor $H$ of a hypersurface $M$ in $\mathbb{E}_{s}^{n+1}, n \geq 4$, were introduced and studied in [6].

6. Quasi-Einstein manifolds. A semi-Riemannian manifold $(M, g), n \geq 2$, is said to be an Einstein manifold if on $M$ we have

$$
S=\frac{\kappa}{n} g
$$

where $S$ and $\kappa$ denote the Ricci tensor and the scalar curvature of $(M, g)$, respectively. According to [10] (see p. 432), (24) is called the Einstein metric condition. It is well known that Einstein manifolds form a natural subclass of various classes of semi-Riemannian manifolds which are determined by curvature conditions imposed on their Ricci tensor ([10], Table, pp. 432-433). For instance, every Einstein manifold belongs to the class of semi-Riemannian manifolds $(M, g)$ satisfying

$$
\nabla\left(S-\frac{\kappa}{2(n-1)} g\right)(X, Y ; Z)=\nabla\left(S-\frac{\kappa}{2(n-1)} g\right)(X, Z ; Y),
$$

for all $X, Y, Z \in \Xi(M)$. (25) means that the tensor $S-\frac{\kappa}{2(n-1)} g$ is a Codazzi tensor on $M$. Semi-Riemannian manifolds of dimensions $\geq 4$ fulfilling (25) are called manifolds with harmonic Weyl tensor ([10], p. 440). We also say that (25) is a generalized Einstein metric condition ([10], Chapter XVI). We note that (16) is also a condition of this type. For a presentation of such conditions we refer to Chapter XVI of [10]. As we show in the next section, some pseudosymmetry type curvature conditions give rise to new examples of curvature conditions of this kind.

A semi-Riemannian manifold $(M, g), n \geq 3$, is called a quasi-Einstein manifold if at every point of $M$ its Ricci tensor $S$ has the following form:

$$
S=\alpha g+\beta w \otimes w, \quad w \in T_{x}^{*} M, \quad \alpha, \beta \in \mathbb{R} .
$$

Evidently, Einstein manifolds is formed by a subclass of quasi-Einstein manifolds. Another subclass of quasi-Einstein manifolds is formed by Ricci-simple manifolds, i.e. manifolds 
having the Ricci tensor of rank at most one. In addition, we mention that semisymmetric Ricci-simple hypersurfaces in semi-Euclidean spaces were investigated in [21]. Examples of such hypersurfaces are also given in [21]. We refer to [27] and [28] for a wider review of results related to the above presentation.

We mention that if (26) is satisfied at $x \in M-U_{H}$ then the Weyl tensor $C$ of a hypersurface $M$ vanishes at $x$ or at this point the Ricci tensor $S$ of $M$ is proportional to the metric tensor ([28], Lemma 4.1(ii)). Therefore we restrict our considerations to the subset $U_{H} \subset M$.

Theorem 6.1 ([28], Theorem 5.1). Let $M$ be a quasi-Einstein hypersurface in $\mathbb{E}_{s}^{n+1}$, $n \geq 4$. Then at every point $x \in U_{H} \subset M$ the following three conditions are equivalent:

$$
\text { (i) } R \cdot S=0, \quad(\text { ii }) \mathcal{A}(W)=0,
$$

$$
\text { (iii) } \mathcal{A}^{3}=\operatorname{tr}(\mathcal{A}) \mathcal{A}^{2}-\frac{\varepsilon \kappa}{n-1} \mathcal{A}, \quad \varepsilon= \pm 1, \quad \alpha=\frac{\kappa}{n-1},
$$

where the vector $W$ is related to $w$ by $g(W, X)=w(X), X \in T_{x} M$, and $w$ and $\alpha$ are defined by (26). Moreover, if at every point $x \in U_{H}$ of $M$ one of the above conditions (i)-(iii) is fulfilled then $\alpha=\frac{\kappa}{n-1}$ and $R \cdot C=Q(S, C)$ at $x$.

We also mention that the above result was applied to the construction of examples of non-semisymmetric Ricci-semisymmetric quasi-Einstein (or non-quasi-Einstein) hypersurfaces in $\mathbb{E}_{s}^{n+1}, n \geq 5$ (see [19] and references therein).

We note that if (22) is satisfied on the subset $U$ of a hypersurface $M$ in $\mathbb{E}_{s}^{n+1}$ then we have $U \subset U_{H} \subset M$. At all points of the set $M-U$, at which $S \neq 0$ and $C \neq 0$, we have the following relation ([22], Theorem 3.1): $R \cdot R=\frac{\kappa}{n-1} Q(g, R)$. There also exist non-semisymmetric manifolds satisfying (22) ([22]). Recently, hypersurfaces satisfying (22) were investigated in [26]. The following was proved:

Theorem 6.2 ([26], Theorem 4.3). If $M$ is a hypersurface in $E_{s}^{n+1}(c), n \geq 4$, satisfying (22), then $L=1$ and

$$
\begin{aligned}
& R \cdot S=0, \quad C \cdot S=0, \quad S=\frac{\kappa}{n-1} g+\beta \omega \otimes \omega, \\
& \mathcal{A}^{3}=\operatorname{tr}(\mathcal{A}) \mathcal{A}^{2}-\frac{\varepsilon \kappa}{n-1} \mathcal{A}, \quad \varepsilon= \pm 1, \quad \alpha=\frac{\kappa}{n-1}, \quad \mathcal{A}(W)=0,
\end{aligned}
$$

hold on the set $U_{L} \subset U \subset M$ of all points of $U$ at which the associated function $L$ is nonzero, where the vector $W$ is related to $w$ by $g(W, X)=w(X), X \in T_{x} M$, and $w$ and $\alpha$ are defined by (26).

7. Certain generalized Einstein metric conditions. Motivated by Theorem 1.1 we introduce a family of curvature conditions. First of all we note that on any Einstein manifold (10) can be also presented in the form

$$
R \cdot C-C \cdot R=\frac{1}{n-1} Q(S, R)=\frac{1}{n-1} Q(S, C)
$$

In view of this, we can investigate curvature properties of non-Einstein and non-conformally flat semi-Riemannian manifolds $(M, g), n \geq 4$, satisfying one of the following conditions: at every point of $M$ the tensors $R \cdot C-C \cdot R$ and $Q(A, T)$ are linearly dependent, 
where $A=g$ or $A=S$ and $T=R$ or $T=C$. Thus we have the following relations related to the above conditions:

$$
\begin{aligned}
& R \cdot C-C \cdot R=L_{1} Q(g, C), \\
& R \cdot C-C \cdot R=L_{2} Q(g, R), \\
& R \cdot C-C \cdot R=L_{3} Q(S, C), \\
& R \cdot C-C \cdot R=L_{4} Q(S, R),
\end{aligned}
$$

where $L_{1}$ is some function on $U_{C}, L_{2}$ some function on $U_{R}, L_{3}$ some function on $U$ defined in Section 1 and $L_{4}$ some function on $U$ defined in Section 5, respectively. Recently, curvature properties of semi-Riemannian manifolds satisfying (29) - (32) are investigated in [24], [27] and [37]. There are also other curvature conditions of this kind ([20], [39]).

Let $(M, g), n \geq 4$, be a semi-Riemannian manifold. In view of Theorem 1.1, we restrict our considerations to the subset $\mathcal{U}=U_{S} \cap U_{C}$ of $M$. It is clear that $\mathcal{U} \subset U_{R}$. The main result related to $(29)$ is given in the following

Theorem 7.1 ([27], Theorem 4.1). Let $(M, g), n \geq 4$, be a semi-Riemannian manifold fulfilling (29). Then $R \cdot R=L_{1} Q(g, R)$ and $C \cdot R=0$ on $\mathcal{U} \subset M$.

Corollary 7.1 ([27], Corollary 5.1). Let $(M, g), n \geq 4$, be a semi-Riemannian manifold fulfilling $R \cdot C=C \cdot R$. Then $R \cdot R=0$ and $C \cdot R=0$ on $\mathcal{U}$.

We also have certain inverse statements (see [27], Proposition 4.2 and Proposition 4.3).

Investigating the condition (30) we considered two cases: the quasi-Einstein case and the non-quasi-Einstein case and we obtained the following results.

Theorem 7.2 ([24], Theorem 3.1). Let $(M, g), n \geq 4$, be a semi-Riemannian manifold satisfying (26) and (30). Then we have the following curvature identities on $\mathcal{U}$ :

$$
S=\beta w \otimes w, \quad \kappa=0, \quad R \cdot R=0, \quad C \cdot R=0, \quad \sum_{X, Y, Z} w(X) \mathcal{C}(Y, Z)=0 .
$$

Theorem 7.3 ([24], Theorem 4.1). Let $(M, g), n \geq 4$, be a non-quasi-Einstein semiRiemannian manifold fulfilling (30). Then on $\mathcal{U}$ the curvature tensor $R$ is of the form (14), where $\mu\left(\mu-\frac{1}{n-2}\right)=\phi \eta$. Consequently, $R \cdot R=0$ on $\mathcal{U}$.

Propositions 3.2 and 4.2 of [24] are inverse statements to the above theorems.

Taking into account Theorem 7.2 and Theorem 7.3 we obtain

Theorem 7.4 ([24], Theorem 4.2). Let $(M, g), n \geq 4$, be a semi-Riemannian manifold fulfilling (30). Then $R \cdot R=0$ on $\mathcal{U}$.

Examples of Ricci-simple as well as non-quasi-Einstein semi-Riemannian manifolds satisfying $R \cdot R=0$ and $C \cdot R=L Q(g, R)$ are given in [38]. Warped products satisfying (30) are described in [24].

The conditions (31) and (32) will be investigated in subsequent papers. We also have some partial results related to $(32)$.

THEOREM 7.5 ([37]). Let $(M, g), n \geq 4$, be a Ricci-semisymmetric manifold fulfilling (32). Then on $\mathcal{U} \cap U_{L}$ we have $S(W, \mathcal{R}(X, Y) Z)=\frac{\kappa}{n-1} R(X, Y, Z, W)$ and $L_{4}=\frac{1}{n-2}$. 
We also have the following inverse statement.

Proposition 7.1 ([37]). Let $(M, g), n \geq 4$, be a semi-Riemannian manifold. If

$$
S(W, \mathcal{R}(X, Y) Z)=\frac{\kappa}{n-1} R(X, Y, Z, W),
$$

then $(M, g)$ is a Ricci-semisymmetric manifold fulfilling (32) with $L_{4}=\frac{\kappa}{n-2}$.

\section{References}

[1] B. E. Abdalla and F. Dillen, A Ricci-semi-symmetric hypersurface of the Euclidean space which is not semi-symmetric, Proc. Amer. Math. Soc. 130 (2002), 1805-1808.

[2] M. A. Akivis and V. V. Goldberg, Conformal Differential Geometry and its Generalizations, Wiley-Interscience Publication, New York, 1996.

[3] M. A. Akivis and V. V. Goldberg, Semiintegrable almost Grassmann structures, Diff. Geom. Appl. 10 (1999), 257-294.

[4] L. Aliás, A. Romero, and M. Sánchez, Compact spacelike hypersurfaces of constant mean curvature in generalized Robertson-Walker spacetimes, in: Geometry and Topology of Submanifolds, VII, World Sci., River Edge, NJ, 1995, 67-70.

[5] K. Arslan, Y. Celik, R. Deszcz, and R. Ezentas, On the equivalence of Ricci-semisymmetry and semisymmetry, Colloq. Math. 76 (1998), 279-294.

[6] K. Arslan, R. Deszcz, R. Ezentaș, C. Murathan, and C. Özgür, On pseudosymmetry type hypersurfaces of semi-Euclidean spaces, I, Acta Math. Sci., in print.

[7] M. Belkhelfa, Differential Geometry of Semi-Riemannian Manifolds and Submanifolds, PhD thesis, Catholic University of Leuven, 2001.

[8] M. Belkhelfa, R. Deszcz, and L. Verstraelen, Pseudosymmetry of 3-dimensional D'Atri spaces, Dept. Math. Agricultural Univ. Wrocław, Ser. A, Theory and Methods, Report No. 86, 2000.

[9] M. Belkhelfa, R. Deszcz, M. Głogowska, D. Kowalczyk, and L. Verstraelen, Some observations on pseudo-symmetry and geodesic symmetry, Dept. Math. Agricultural Univ. Wrocław, Ser. A, Theory and Methods, Report No. 91, 2000.

[10] A. L. Besse, Einstein Manifolds, Ergeb. Math. Grenzgeb. 10, Springer, Berlin, 1987.

[11] B. Y. Chen, A Riemannian invariant for submanifolds in space forms and its applications, in: Geometry and Topology of Submanifolds, VI, World Sci., River Edge, NJ, 1994, 58-81.

[12] F. Defever, R. Deszcz, M. Głogowska, V. V. Goldberg, and L. Verstraelen, A class of four-dimensional warped products, Demonstratio Math., in print.

[13] F. Defever, R. Deszcz, and M. Prvanović, On warped product manifolds satisfying some curvature condition of pseudosymmetry type, Bull. Greek Math. Soc. 36 (1994), 43-67.

[14] F. Defever, R. Deszcz, L. Verstraelen, and L. Vrancken, On pseudosymmetric spacetimes, J. Math. Phys. 35 (1994), 5908-5921.

[15] R. Deszcz, Pseudosymmetry curvature conditions imposed on the shape operators of hypersurfaces in the affine spaces, Results in Math. 20 (1991), 600-621.

[16] R. Deszcz, On pseudosymmetric spaces, Bull. Soc. Math. Belg. Sér. A 44 (1992), 1-34.

[17] R. Deszcz, Certain curvature characterizations of affine hypersurfaces, Colloq. Math. 63 (1992), 21-39.

[18] R. Deszcz, Pseudosymmetric hypersurfaces in spaces of constant curvature, Tensor (N.S.) 58 (1997), 253-269. 
[19] R. Deszcz and M. Głogowska, Examples of nonsemisymmetric Ricci-semisymmetric hypersurfaces, Colloq. Math. 94 (2002), 87-101.

[20] R. Deszcz and M. Głogowska, Some nonsemisymmetric Ricci-semisymmetric warped product hypersurfaces, Publ. Inst. Math. (Beograd), in print.

[21] R. Deszcz, M. Głogowska, M. Hotloś, and Z. Șentürk, On certain quasi-Einstein semisymmetric hypersurfaces, Ann. Univ. Sci. Budapest. Eötvös Sect. Math. 41 (1998), 151-164.

[22] R. Deszcz and M. Hotloś, On a certain extension of the class of semisymmetric manifolds, Publ. Inst. Math. (Beograd) (N.S.) 63(77) (1998), 115-130.

[23] R. Deszcz and M. Hotloś, On a certain subclass of pseudosymmetric manifolds, Publ. Math. Debrecen 53 (1998), 29-48.

[24] R. Deszcz and M. Hotloś, On some condition of pseudosymmetry type, Tsukuba J. Math., in print.

[25] R. Deszcz, M. Hotloś, and Z. Șentürk, On a certain application of the Patterson's curvature equality, Publ. Math. Debrecen 58 (2001), 93-107.

[26] R. Deszcz, M. Hotloś, and Z. Șentürk, Quasi-Einstein hypersurfaces in semi-Riemannian space forms, Colloq. Math. 89 (2001), 81-97.

[27] R. Deszcz, M. Hotloś, and Z. Șentürk, On some family of generalized Einstein metric conditions, Demonstratio Math. 34 (2001), 943-954.

[28] R. Deszcz, M. Hotloś, and Z. Șentürk, On curvature properties of quasi-Einstein hypersurfaces in semi-Euclidean spaces, Soochow J. Math. 27 (2001), 375-389.

[29] R. Deszcz, P. Verheyen, and L. Verstraelen, On some generalized Einstein metric conditions, Publ. Inst. Math. (Beograd) (N.S.) 60(74) (1996), 108-120.

[30] R. Deszcz, L. Verstraelen, and L. Vrancken, On the symmetry of warped product spacetimes, Gen. Relativity Gravitation 23 (1991), 671-681.

[31] R. Deszcz, L. Verstraelen, and S.. Yaprak, On 2-quasi-umbilical hypersurfaces in conformally flat spaces, Acta Math. Hung. 78 (1998), 45-57.

[32] R. Deszcz and Ș. Yaprak, Curvature properties of Cartan hypersurfaces, Colloq. Math. 67 (1994), 91-98.

[33] R. Deszcz and S.. Yaprak, Curvature properties of certain pseudosymmetric manifolds, Publ. Math. Debrecen 45 (1994), 333-345.

[34] M. Głogowska, Curvature properties of some four-dimensional manifolds, Demonstratio Math. 34 (2001), 900-918.

[35] M. Głogowska, On a curvature characterization of Ricci-pseudosymmetric hypersurfaces, Dept. Math., Agricultural Univ. Wrocław, Ser. A, Theory and Methods, Report No. 97, 2001.

[36] N. Hashimoto and M. Sekizawa, Three-dimensional conformally flat pseudo-symmetric spaces of constant type, Arch. Math. (Brno) 36 (2000), 279-286.

[37] M. Hotloś, On certain curvature condition of pseudosymmetry type, to appear.

[38] D. Kowalczyk, On semi-Riemannian manifolds satisfying some curvature conditions, Soochow J. Math. 27 (2001), 445-461.

[39] D. Kowalczyk, On the Schwarzschild type spacetime, Dept. Math., Agricultural Univ. Wrocław, Ser. A, Theory and Methods, Report No. 83, 2000.

[40] O. Kowalski and M. Sekizawa, Pseudo-symmetric spaces of constant type in dimension three-elliptic spaces, Rend. Mat. Appl. 17 (1997), 477-512.

[41] O. Kowalski and M. Sekizawa, Pseudo-symmetric spaces of constant type in dimension three-non-elliptic spaces, Bull. Tokyo Gakugei Univ. 50 (1998), 1-28. 
[42] C. Murathan, K. Arslan, R. Deszcz, R. Ezentaṣ, and C. Özgür, On some class of hypersurfaces of semi-Euclidean spaces, Publ. Math. Debrecen 2001 (2001), 587-604.

[43] K. Nomizu, On hypersurfaces satisfying a certain condition on the curvature tensor, Tôhoku Math. J. 20 (1968), 46-59.

[44] W. Roter, On the existence of certain conformally recurrent metrics, Colloq. Math. 51 (1987), 315-327.

[45] M. Sánchez, On the geometry of generalized Robertson-Walker spacetimes: geodesics, Gen. Relativity Gravitation 30 (1998), 915-932.

[46] Z. I. Szabó, Structure theorems on Riemannian spaces satisfying $R(X, Y) \cdot R=0$. I. The local version, J. Diff. Geom. 17 (1982), 531-582.

[47] H. Takagi, An example of a Riemannian manifold satisfying $R(X, Y) \cdot R=0$ but not $\nabla R=0$, Tôhoku Math. J. 24 (1972), 105-108.

[48] L. Verstraelen, Comments on pseudo-symmetry in the sense of Ryszard Deszcz, in: Geometry and Topology of Submanifolds, VI, World Sci., River Edge, NJ, 1994, 199-209. 